\title{
Significance of cancer stroma for bone destruction in oral squamous cell carcinoma using different cancer stroma subtypes
}

\author{
QIUSHENG SHAN $^{1,2}$, KIYOFUMI TAKABATAKE ${ }^{1}$, HOTAKA KAWAI ${ }^{1}$, MAY WATHONE OO ${ }^{1}$, YASUNORI INADA ${ }^{1}$, \\ SHINTARO SUKEGAWA ${ }^{3}$, SHIGEKO FUSHIMI ${ }^{1}$, KEISUKE NAKANO ${ }^{1}$ and HITOSHI NAGATSUKA ${ }^{1}$ \\ Departments of ${ }^{1}$ Oral Pathology and Medicine and ${ }^{2}$ Oral and Maxillofacial Surgery, \\ Okayama University Graduate School of Medicine, Dentistry and Pharmaceutical Sciences, Okayama 700-8525; \\ ${ }^{3}$ Department of Oral and Maxillofacial Surgery, Kagawa Prefectural Central Hospital, Takamatsu, Kagawa 760-8557, Japan
}

Received November 15, 2021; Accepted February 7, 2022

DOI: $10.3892 /$ or.2022.8292

\begin{abstract}
Stromal cells in the tumor microenvironment (TME) can regulate the progression of numerous types of cancer; however, the bone invasion of oral squamous cell carcinoma (OSCC) has been poorly investigated. In the present study, the effect of verrucous SCC-associated stromal cells (VSCC-SCs), SCC-associated stromal cells (SCC-SCs) and human dermal fibroblasts on bone resorption and the activation of HSC-3 osteoclasts in vivo were examined by hematoxylin and eosin, AE1/3 (pan-cytokeratin) and tartrate-resistant acid phosphatase staining. In addition, the expression levels of matrix metalloproteinase (MMP)9, membrane-type 1 MMP (MT1-MMP), Snail, receptor activator of $\mathrm{NF}-\kappa \mathrm{B}$ ligand (RANKL) and parathyroid hormone-related peptide (PTHrP) in the bone invasion regions of HSC-3 cells were examined by immunohistochemistry. The results suggested that both SCC-SCs and VSCC-SCs promoted bone resorption, the activation of osteoclasts, and the expression levels of MMP9, MT1-MMP, Snail, RANKL and PTHrP. However, SCC-SCs had a more prominent effect compared with VSCC-SCs. Finally, microarray data were used to predict potential genes underlying the differential effects of VSCC-SCs and SCC-SCs on bone invasion in OSCC. The results revealed that IL1B, ICAM1, FOS, CXCL12, INS and NGF may underlie these differential effects. In conclusion, both VSCC-SCs and SCC-SCs may promote bone invasion in OSCC by enhancing the expression levels of RANKL in cancer and stromal cells mediated by PTHrP; however, SCC-SCs had a more prominent effect. These findings may
\end{abstract}

Correspondence to: Dr Kiyofumi Takabatake, Department of Oral Pathology and Medicine, Okayama University Graduate School of Medicine, Dentistry and Pharmaceutical Sciences, 2-5-1 Shikata-cho, Kita-ku, Okayama 700-8525, Japan

E-mail: gmd422094@s.okayama-u.ac.jp

Key words: oral squamous cell carcinoma, bone invasion, osteoclast, receptor activator of $\mathrm{NF}-\kappa \mathrm{B}$ ligand, parathyroid hormone-related peptide, microarray, cancer-associated stromal cells represent a potential regulatory mechanism underlying the bone invasion of OSCC.

\section{Introduction}

Oral squamous cell carcinoma (OSCC) is the most common type of malignant tumor in the head and neck region. It has a high rate of recurrence and distant metastasis, and can also invade into nearby bone tissues, such as the maxilla and mandible, resulting in bone destruction and difficult treatment of OSCC (1-4). Bone invasion in OSCC is mediated by osteoclasts rather than cancer cells. Osteoclastogenesis is regulated by receptor activator of $\mathrm{NF}-\kappa \mathrm{B}(\mathrm{RANK}), \mathrm{RANK}$ ligand (RANKL) and osteoprotegerin, which belong to the tumor necrosis factor (TNF) family (5-7). Cancer-induced bone destruction is regulated by various factors that are synthesized by cancer cells, including parathyroid hormone-related peptide (PTHrP), interleukin (IL)-6, IL-11, TNF- $\alpha$ and prostaglandin E2, which are also synthesized by OSCC cells (8-11). These factors stimulate RANKL expression in stromal and osteoblastic cells adjacent to the resorbing bone. IL- 6 produced by stromal cells in OSCC can induce fibroblastic stromal cells to produce RANKL (12). Therefore, it is necessary to study the potential mechanisms by which OSCC invades bone tissue.

Solid tumors consist of parenchymal and stromal areas, and the crosstalk between them serves a crucial role in the progression of cancer (13). Stromal cells in the tumor microenvironment (TME) mainly consist of cancer-associated fibroblasts (CAFs), tumor-associated macrophages and immune cells, which have a significant role in the invasion and metastasis of cancer (14). The highly expressed C-X-C motif chemokine ligand 1 (CXCL1) in CAFs has been reported to promote the invasion of the poorly-differentiated oral cancer cell line SAS (15). In addition, the Axin2-Snail axis may promote the activation of CAFs in the TME, which in turn can enhance the bone invasion of OSCC (16). Compared with in normal fibroblasts, the overexpression of RANKL in CAFs may activate osteoclasts, resulting in the bone invasion of OSCC. In addition, CAFs can induce macrophage transfer into osteoclasts by co-culture with macrophages (17). Therefore, stromal cells in the TME could regulate the bone invasion of OSCC by crosstalk with cancer cells. However, to the best of 
our knowledge, the details of how the tumor stroma influences bone resorption in cancer cells remain unknown.

The differences between the macroscopic subtypes of OSCC are defined by cancer parenchyma properties. OSCC is divided into the endophytic (ED)-type and exophytic (EX)-type according to its invasive abilities. ED-type OSCC is invasive and can occasionally metastasize. Conversely, EX-type OSCC, such as verrucous OSCC, presents an outward growth, does not invade the subepithelial connective tissue and does not metastasize (18-20). We previously reported that the cancer stroma regulates the biological characteristics of cancer parenchyma. In addition, EX-type verrucous SCC-associated stromal cells (VSCC-SCs) and ED-type SCC-associated stromal cells (SCC-SCs) were shown to have different effects on bone invasion, migration and differentiation of HSC-2 and HSC-3 cells. Furthermore, the expression levels of $\alpha$-SMA in SCC-SCs were revealed to be higher than those in VSCC-SCs. Apart from this, the detailed different marker profiles between VSCC-SCs and SCC-SCs were analyzed using microarray data $(21,22)$. However, these previous studies only identified the effects of VSCC-SCs and SCC-SCs on bone invasion of OSCC and did not assess the potential regulatory mechanisms. The present study aimed to investigate the detailed phenomenon and potential regulatory mechanisms by which VSCC-SCs and SCC-SCs regulate the bone invasion of OSCC using tumor stroma established from patients with OSCC with different degrees of invasiveness. For this purpose, HSC-3 cells were selected as a cell model due to the fact that HSC-3 is a poorly-differentiated oral cancer cell line with obvious bone invasion ability that is widely used in bone invasion research $(12,23)$. Human dermal fibroblasts (HDFs) were selected as a negative control, mainly because HDFs are normal fibroblasts that are not edited by cancer cells $(21,22)$. VSCC-SCs and SCC-SCs were extracted from patients with OSCC to examine their effects. These findings highlight the potential regulatory mechanism underlying bone invasion in OSCC.

\section{Materials and methods}

Cells and cell culture. The human oral cancer cell line HSC-3 was purchased from the Japanese Collection of Research Bioresources Cell Bank. HDFs (cat. no. CC-2511) were purchased from Lonza Group, Ltd. The murine macrophage cell line RAW264.7 was obtained from the RIKEN BioResource Center. VSCC-SCs and SCC-SCs were extracted from surgical operative tissues at the Department of Oral and Maxillofacial Surgery, Okayama University Graduate School of Medicine, Dentistry and Pharmaceutical Sciences (Okayama, Japan). The VSCC tissues were obtained from one patient with VSCC and SCC tissues were obtained from one patient with SCC (mean age, 81 years; sex of patients, both female), to separate the stromal cells and generate the cell culture. Sections of fresh OSCC tissue $\left(1 \mathrm{~mm}^{3}\right)$ were washed several times with $\alpha$-Modified Eagle's medium ( $\alpha$-MEM; Thermo Fisher Scientific, Inc.) containing antibiotic-antimycotic (Thermo Fisher Scientific, Inc.) and then minced. The tissues were then treated with $\alpha$-MEM containing $1 \mathrm{mg} / \mathrm{ml}$ collagenase II (Invitrogen; Thermo Fisher Scientific, Inc.) and dispase (Invitrogen; Thermo Fisher Scientific, Inc.) for $2 \mathrm{~h}$ at $37^{\circ} \mathrm{C}$ with agitation $(200 \mathrm{rpm})$. The released cells were centrifuged for $5 \mathrm{~min}$ at $111.8 \mathrm{x} \mathrm{g}$ at room temperature, suspended in $\alpha$-MEM containing 10\% FBS (Biowest), filtered through a cell strainer (100 $\mu \mathrm{m}$; Falcon; Corning Life Sciences), plated in a tissue culture flask and incubated at $37^{\circ} \mathrm{C}$ in an atmosphere containing $5 \% \mathrm{CO}_{2}$. After 1 week, the stromal cells were separated by Accutase (Invitrogen; Thermo Fisher Scientific, Inc.) based on the different adhesive properties of epithelial and stromal cells $(21,22)$. HSC-3, VSCC-SCs, SCC-SCs and HDFs were maintained in $\alpha$-MEM supplemented with $10 \% \mathrm{FBS}$ and $1 \%$ antimycotic-antibiotic at $37^{\circ} \mathrm{C}$ in a humidified atmosphere containing $5 \% \mathrm{CO}_{2}$ and $95 \%$ air. The present study was approved by the Ethics Committee of Okayama University (project identification code: 1703-042-001). In addition, written informed consent was obtained from all patients.

Tartrate-resistant acid phosphatase (TRAP) staining of cells. RAW264.7, HSC-3, VSCC-SCs, SCC-SCs and HDFs were digested with Accutase and EDTA (Thermo Fisher Scientific, Inc.), and centrifuged at $111.8 \mathrm{x} \mathrm{g}$ for $5 \mathrm{~min}$ at room temperature when the density approached 90\%. RAW264.7 cells were mixed with or without VSCC-SCs/SCC-SCs/HDFs and HSC-3 at a 3:3:1 ratio. The mixed cells were seeded into a 6-well plate containing coverslips $(22 \times 22 \mathrm{~mm}$; Matsunami) at a density of $3.5 \times 10^{5} /$ well. After incubation for 3 days $\left(37^{\circ} \mathrm{C}\right)$, the attached slides were washed three times with TBS and were then fixed with $4 \%$ paraformaldehyde for $15 \mathrm{~min}$. After washing with TBS for a further three times, the slides were stained using a TRAP staining kit (cat. no. AK04F; Cosmo Bio Co., Ltd.) according to the manufacturer's instructions in $37^{\circ} \mathrm{C}$ incubator. Images of the slides were captured using a bright-field microscope (magnifications, x4, x10, x20 and x40; BX51; Olympus Corporation). A total of five images (magnification, $\mathrm{x} 40$ ) were randomly captured to calculate the percentage of positive osteoclast cells (percentage of TRAP-positive cells) using Image J software (version 1.53K; National Institutes of Health).

Experimental animals. All animal experiments were conducted according to the relevant guidelines and regulations approved by the institutional committees at Okayama University (approval no. OKU-2017406). The anesthesia protocol was performed in accordance with Laboratory Animal Anesthesia, 3rd edition (24). To confirm mice were appropriately anesthetized, the mice were checked to determine whether they would return to a prone position when they were placed on their backs. Following intraperitoneal anesthesia with ketamine hydrochloride $(75 \mathrm{mg} / \mathrm{kg}$ body weight) and medetomidine hydrochloride $(0.5 \mathrm{mg} / \mathrm{kg}$ body weight), $200 \mu 1$ mixed cells including HSC-3 $\left(1 \times 10^{6}, 100 \mu 1\right)$ and stromal cells (VSCC-SCs, SCC-SCs and HDFs; $3 \times 10^{6}, 100 \mu \mathrm{l}$ ) were injected into the subcutaneous tissue in the central region of the top of the head of 20 healthy female BALB-c nu-nu mice (age, 4 weeks; mean weight, 15 g; Shimizu Laboratory Supplies Co., Ltd) gradually and slowly (16). All mice were reared in an animal room at a temperature of $25^{\circ} \mathrm{C}$ with $50-60 \%$ humidity under a 12-h light/dark cycle. All mice were allowed free access to food and water. The experimental animals were divided into the HSC-3, HSC-3 + VSCC-SCs, HSC-3 + SCC-SCs and HSC-3 + HDFs groups (n=5/group). Of the five mice, one mouse in each group exhibited poor tumor 
formation; therefore, their data were removed and the data from the remaining four mice were used for data analysis.

Hematoxylin and eosin $(H \& E)$ staining. After 4 weeks, all mice were sacrificed by excess inhalation of isoflurane (concentration $>5 \%$ ). Cardiac arrest was verified by pulse palpation followed by cervical dislocation. The whole heads of mice containing the tumor and bone tissues were removed, fixed in $4 \%$ paraformaldehyde for $12 \mathrm{~h}$ and decalcified with $10 \%$ EDTA for 4 weeks at $4^{\circ} \mathrm{C}$. Subsequently, the whole heads of animal models were processed and embedded into paraffin wax through routine histological preparation and further cut into $5-\mu \mathrm{m}$ sections. Finally, the sections were used for H\&E staining. The sections were stained with Carrazi's hematoxylin (Muto Pure Chemicals Co., Ltd.) for $5 \mathrm{~min}$ at room temperature and stained with eosin (Muto Pure Chemicals Co., Ltd.) for $7 \mathrm{~min}$ at room temperature. Images of the bone invasion regions of tissues were captured using a bright-field microscope (magnification, $\mathrm{x} 40$ ).

TRAP staining of tissue. The 5- $\mu \mathrm{m}$ sections of tissue samples from the HSC-3, HSC-3 + VSCC-SCs, HSC-3 + SCC-SCs and HSC-3 + HDF groups were used for TRAP staining using the aforementioned TRAP staining kit according to the manufacturer's instructions, and active multi-nucleated osteoclasts on the bone surface were considered to be TRAP-positive cells. The bone invasion regions of tissues were photographed using a bright-field microscope. A total of five images (magnification, x40; BX51; Olympus Corporation) were randomly captured from each mouse to obtain positive cell counts using ImageJ software (version 1.53K).

Immunohistochemistry (IHC). Following antigen retrieval in a microwave for 1 or $8 \mathrm{~min}$ in $0.01 \mathrm{M}$ tri-sodium citrate buffer ( $\mathrm{pH}$ 6) and 0.01 M Dako Target Retrieval Solution ( $\mathrm{pH} 9$; cat. no. S2367; Agilent Technologies, Inc.), 5- $\mu \mathrm{m}$ sections were blocked with $10 \%$ normal serum (Vector Laboratories, Inc.) for $20 \mathrm{~min}$ at room temperature and incubated with primary antibodies, including mouse anti-pan-cytokeratin (AE1/3; cat. no. ab27988; 1:20; Abcam), anti-matrix metalloproteinase (MMP)9 (F-69; 1:20; Kyowa Pharma Chemical Co., Ltd.) anti-membrane-type 1 MMP (MT1-MMP; F-86; 1:20; Kyowa Pharma Chemical Co., Ltd.), rabbit anti-Snail + SLUG (cat. no. ab180714; 1:200; Abcam), rabbit anti-RANKL (cat. no. bs-0747R; 1:100; Bioss) and anti-PTHrP (cat. no. 10817-1-AP; 1:100; Proteintech Group Inc.) overnight at $4^{\circ} \mathrm{C}$. After washing three times with TBS, all sections were incubated with secondary antibody avidin-biotin complexes [mouse (cat. no. PK-6102)/rabbit (cat. no. PK-6101) ABC kit; blocking serum (normal serum, diluted with TBS), 1:75; biotinylated secondary antibody (diluted with normal serum), 1:200; reagent A (Avidin, ABC Elite) and reagent B (biotinylated HRP, ABC Elite, diluted by TBS), 1:55; Vector Laboratories, Inc.] for $1 \mathrm{~h}$ at room temperature followed by visualization with diaminobenzidine (DAB) $/ \mathrm{H}_{2} \mathrm{O}_{2}$ mixed solution (Histofine DAB substrate; Nichirei Biosciences, Inc.). Images of the erosive area of the bone invasion region in the tissues were captured using a bright-field microscope. A total of ten images (magnification, $\mathrm{x} 40$ ) were acquired for each mouse to assess the expression levels of MMP9, MT1-MMP, Snail,
RANKL and PTHrP by IHC score. IHC score was calculated as follows: Percentage of positive cells score $\mathrm{x}$ intensity score. The percentage of positive cells score was defined as: $0(<1 \%)$; 1 (1-24\%); 2 (25-49\%); 3 (50-74\%); 4 (75-100\%). The intensity score was defined as: 0 , no staining; 1 , light yellow color (weak staining); 2, brown color (moderate strong staining); and 3 , dark brown color (strong staining) (25).

Microarray and bioinformatics analyses. RNA was extracted from cultured VSCC-SCs and SCC-SCs to conduct microarray. RNA was first extracted by RNeasy mini spin columns (Qiagen, Inc.) and the quality was checked using a NanoDrop (NanoDrop; Thermo Fisher Scientific, Inc.) and BioAnalyzer (Agilent Technologies, Inc.). Subsequently, cDNA synthesis, cRNA labeling and amplification processed were conducted using the Low Input Quick Amp Labeling Kit (Agilent Technologies, Inc.), and the purification of labeled cRNAs was conducted using RNeasy mini spin columns (Qiagen, Inc.). Finally, the microarray (SurePrint G3 Human 8x60K ver.3.0; Agilent Technologies, Inc.) was scanned using a G2505C Microarray Scanner (Agilent Technologies, Inc.) and bioinformatics analyses were performed. The GeneSpring GX 14.9 was used to determine the differentially expressed genes (DEGs) in SCC-SCs compared with in VSCC-SCs. $\mid \mathrm{LogFCl}>1$ was considered as the cut-off value (the dataset was uploaded to the Gene Expression Omnibus database, https://www.ncbi.nlm. nih.gov/geo/query/acc.cgi?\&acc=GSE164374). The upregulated DEGs that interact with MMP9, MT1-MMP, Snail, RANKL and PTHrP were examined by protein-to-protein interaction network (PPI) analysis using STRING (http://string-db.org/) and Cytoscape 3.7.2 (cytohubba; https://cytoscape.org/). A combined score $>0.4$ was considered as the cut-off value and the hub genes were selected according to the degree. The hub genes that were differentially expressed in SCC-SCs compared with in VSCC-SCs were analyzed by heatmap (a software plug-in within SangerBox; http://sangerbox.com). Finally, the differentially expressed hub genes that interact with MMP9, MT1-MMP, Snail, RANKL and PTHrP were depicted in a Venn plot.

Statistical analysis. Statistical analysis was conducted using GraphPad Prism 9 (GraphPad Software, Inc.). The cell experiments were repeated three times, and the animal research was repeated on five independent mice. The parametric data are presented as the mean \pm SD. One-way ANOVA was used to compare the differences among $>2$ groups followed by Tukey's post hoc test. The non-parametric data are presented as the median and IQR. The Kruskal-Wallis test was used to analyze the non-parametric data followed by Dunn's test. $\mathrm{P}<0.05$ was considered to indicate a statistically significant difference.

\section{Results}

Both VSCC-SCs and SCC-SCs promote the activation of osteoclasts in vitro. HSC-3 cells were selected as a cell model due to the fact that HSC-3 is a type of poorly-differentiated oral cancer cell line with obvious bone invasive ability that is widely used in bone invasion research. The RAW264.7 cell line was selected as a cell model as it is comprised of murine macrophages and is widely used in the research of bone invasion research (17). Firstly, TRAP staining was used to determine 
A
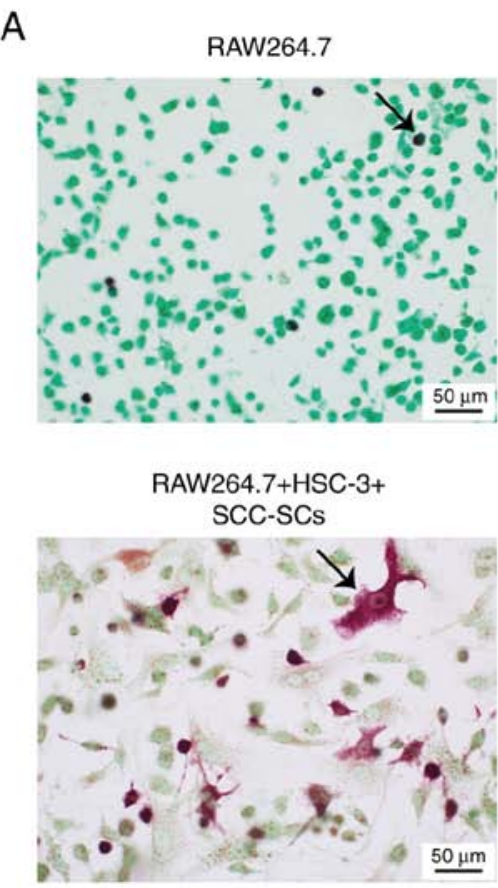

RAW264.7+HSC-3

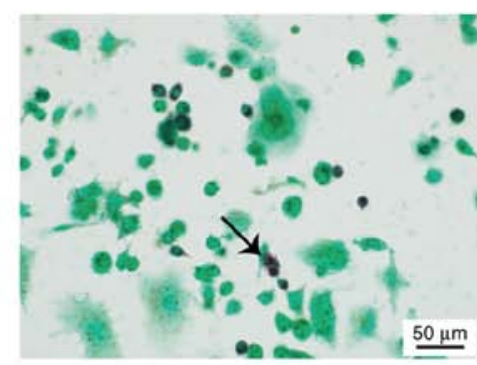

RAW264.7+HSC-3+ HDFs

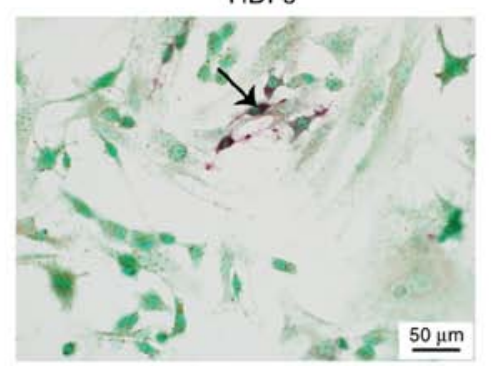

RAW264.7+HSC-3+ VSCC-SCs

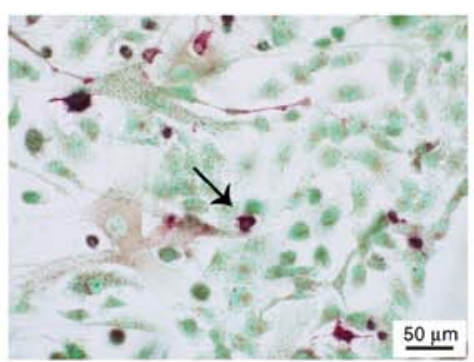

$\mathrm{B}$

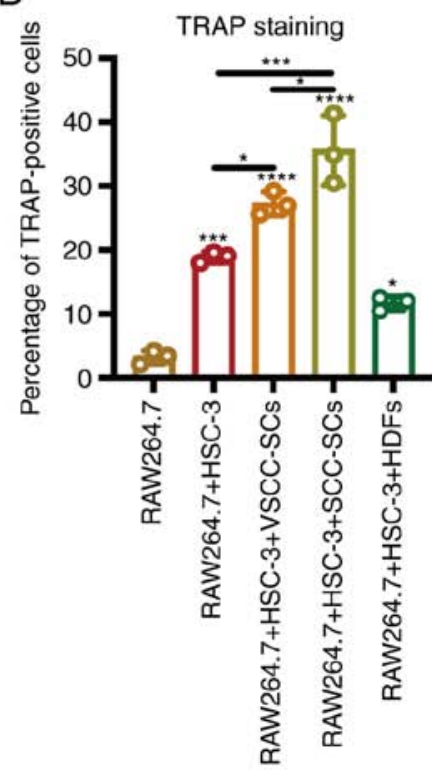

Figure 1. Effects of VSCC-SCs, SCC-SCs and HDFs on the activation of osteoclasts in vitro. (A) TRAP staining was used to test the effects of VSCC-SCs, SCC-SCs and HDFs on the activation of osteoclasts. The arrows indicate the TRAP-positive cells. (B) Semi-quantification of the percentage of positive osteoclasts in different groups of RAW264.7 cells. Data are shown as the mean \pm SD of three independent experiments. Statistical analysis was performed by one-way ANOVA followed by Tukey's post hoc test. ${ }^{*} \mathrm{P}<0.05,{ }^{* * *} \mathrm{P}<0.001,{ }^{* * * *} \mathrm{P}<0.0001$ vs. RAW264.7 or as indicated. HDFs, human dermal fibroblasts; SCC-SC, squamous cell carcinoma-associated stromal cells; TRAP, tartrate-resistant acid phosphatase; VSCC-SC, verrucous squamous cell carcinomaassociated stromal cells.

the percentage of TRAP-positive cells in different groups to determine the effects of VSCC-SCs, SCC-SCs and HDFs on osteoclast activation (Fig. 1). The percentage of TRAP-positive cells was highest in the RAW264.7 + HSC-3 + SCC-SCs group, followed by the RAW264.7 + HSC-3 + VSCC-SCs group. The percentage of TRAP-positive cells in the RAW264.7 + HSC-3 group was slightly higher than that in the RAW264.7 + HSC-3 + HDFs group, whereas it was markedly higher than that in the RAW264.7 group (Fig. 1B). In addition, the RAW264.7 and RAW264.7 + HSC-3 groups mainly contained round-shaped TRAP-positive cells; the RAW264.7 + HSC-3 + VSCC-SCs group mainly contained round-shaped TRAP-positive cells, but with several triangle- and spindle-shaped TRAP-positive cells; the RAW264.7 + HSC-3 + SCC-SCs group mainly contained triangle- and spindle-shaped TRAP-positive cells and few round-shaped TRAP-positive cells; and the RAW264.7 + HSC-3 + HDFs group mainly contained spindleand round-shaped TRAP-positive cells (Fig. 1A). Finally, the sizes of the TRAP-positive cells in the RAW264.7+ HSC-3 + SCC-SCs group were slightly larger than those in the RAW264.7 + HSC-3 + VSCC-SCs group, and markedly larger than those in the RAW264.7, RAW264.7 + HSC-3 and
RAW264.7 + HSC-3 + HDFs groups. There was little difference between the RAW264.7, RAW264.7 + HSC-3 and RAW264.7 + HSC-3 + HDFs groups (Fig. 1A). These findings indicated that both VSCC-SCs and SCC-SCs promoted the activation of osteoclasts by regulating their number, shape and size.

Effects of VSCC-SCs and SCC-SCs on the bone resorption of $H S C-3$ cells in vivo. $\mathrm{H} \& \mathrm{E}$ and $\mathrm{AE} 1 / 3$ (pan-cytokeratin) staining were used to determine the effects of VSCC-SCs, SCC-SCs and HDFs on the bone resorption of HSC-3 cells in vivo. Notably, $\mathrm{AE} 1 / 3$ (pan-cytokeratin) staining is used to detect cancer cells. Histologically, three types of bone invasion are recognized: Erosive, infiltrative and mixed. The erosive type of bone invasion is associated with a sharp transition between the cancer and bone, osteoclastic bone resorption, fibrosis along the cancer and the absence of bone islets within the cancer. The infiltrative type of bone invasion is associated with the formation of irregular nests and projections of cancer cells into the bone, and the presence of residual bone islets inside the cancer. The mixed type includes both types of invasion in the cancer invasion region $(26,27)$. In each group, some regions exhibited the stroma between the cancer and bone area, while some regions 
A

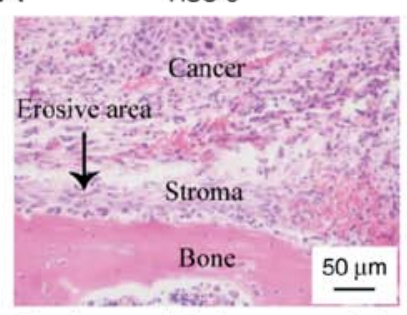

Erosive area (little bone resorptio

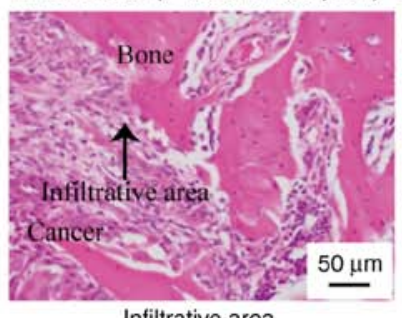

Infiltrative area

B

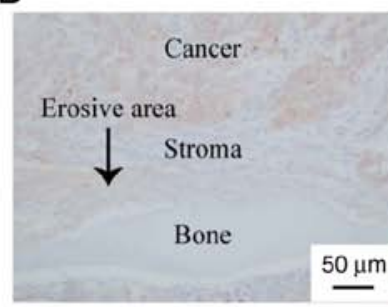

Erosive area

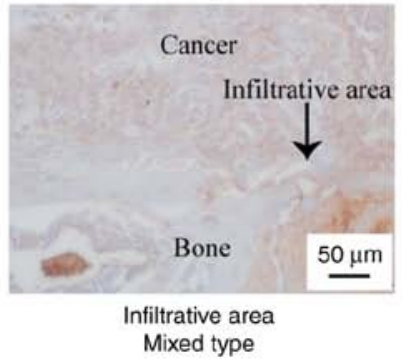

HSC-3+VSCC-SCs
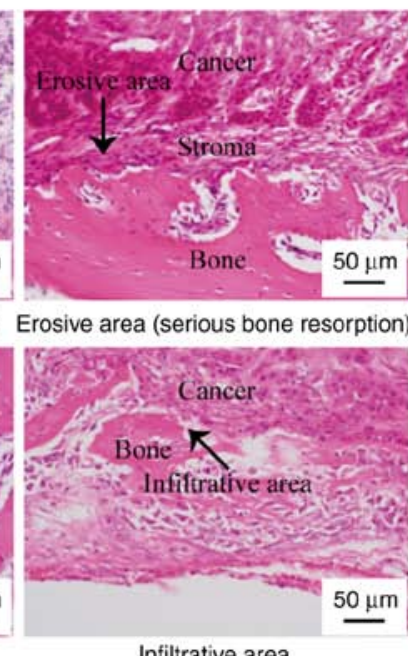

Infiltrative area

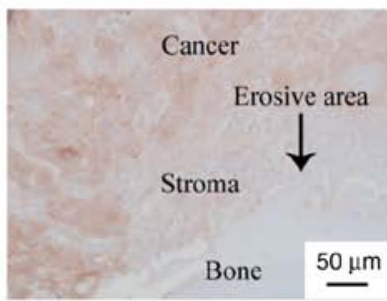

Erosive area

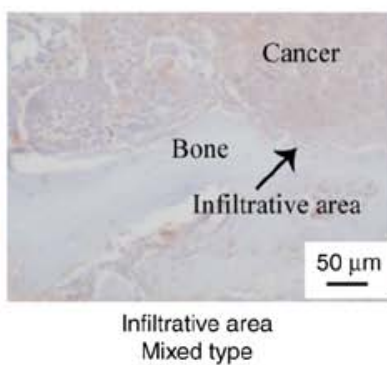

HSC-3+SCC-SCs

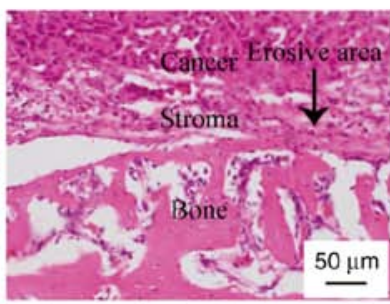

Erosive area (serious bone resorption)

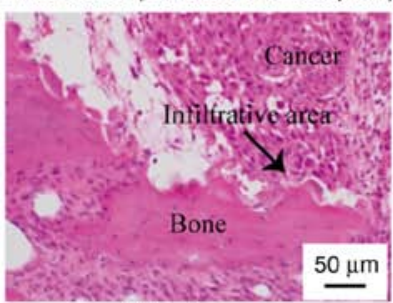

Infiltrative area

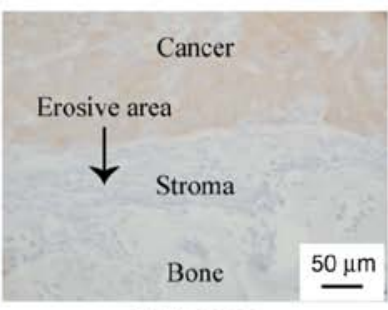

Erosive area

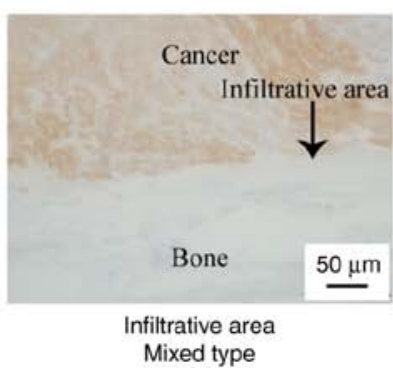

HSC-3+HDFs
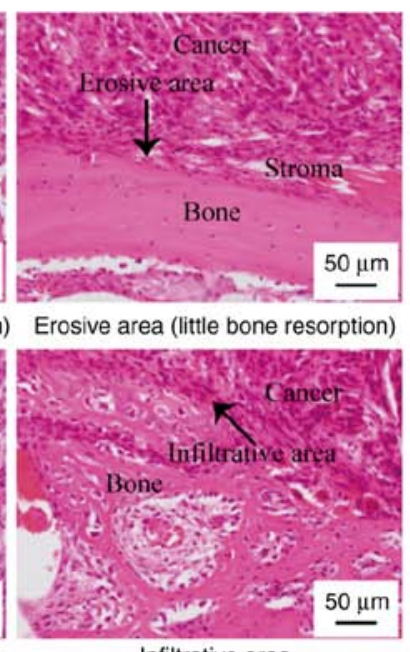

Infiltrative area

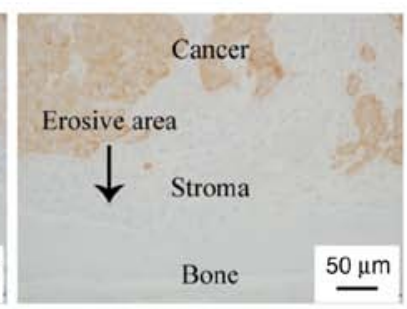

Erosive area

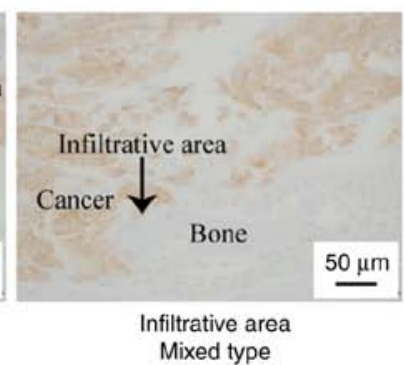

Figure 2. Effects of VSCC-SCs, SCC-SCs and HDFs on the bone resorption of HSC-3 cells in vivo using (A) hematoxylin and eosin, and (B) AE1/3 (pan-cytokeratin) staining. The arrows indicate the erosive area. HDFs, human dermal fibroblasts; SCC-SC, squamous cell carcinoma-associated stromal cells; VSCC-SC, verrucous squamous cell carcinoma-associated stromal cells.

did not contain the stroma between the cancer region and bone region. The results revealed that the four groups exhibited a mixed type of bone invasion (Fig. 2A and B). In both erosive and infiltrative areas, the bone resorption degree of HSC-3 + SCC-SCs was the most serious according to bone resorption morphology, followed by HSC-3 + VSCC-SCs. There was little difference between the HSC-3 and HSC-3 + HDFs groups (Fig. 2A). These data indicated that both VSCC-SCs and SCC-SCs promoted the bone resorption of HSC-3 in vivo, and SCC-SCs had a more prominent effect than VSCC-SCs.

Both VSCC-SCs and SCC-SCs promote osteoclast activation in the bone invasion regions of HSC-3 cells in vivo. TRAP staining was used to determine the number of active, multi-nucleated osteoclasts to determine the effects of VSCC-SCs, SCC-SCs and HDFs on the bone invasion regions of HSC-3 cells in vivo. The sizes of giant osteoclasts in the HSC-3 + SCC-SCs group were slightly larger than those in the HSC-3 + VSCC-SCs and HSC-3 groups, whereas they were markedly larger than those in the HSC-3 + HDFs group in both erosive and infiltrative areas (Fig. 3A). In addition, the number of active multi-nucleated osteoclasts in the erosive area in the HSC-3 + SCC-SCs group was slightly higher than that in HSC-3 + VSCC-SCs group, whereas it was markedly higher than those in the HSC-3 and HSC-3 + HDFs groups in both erosive and infiltrative areas; all of these comparisons in the erosive area were significantly different (Fig. 3A and B). In erosive areas, the HSC-3 + SCC-SCs group mainly contained triangle-shaped osteoclasts, and the HSC-3 + VSCC-SCs and HSC-3 groups contained triangle-, ellipse- and round-shaped osteoclasts, whereas there were only ellipse-shaped osteoclasts in the HSC-3 + HDFs group (Fig. 3A). In infiltrative areas, the HSC-3 + SCC-SCs group mainly contained round-shaped osteoclasts and the HSC-3 + VSCC-SCs group contained triangle-, ellipse- and round-shaped osteoclasts, whereas there were ellipse- and round-shaped osteoclasts in the HSC-3 and HSC-3 + HDFs groups (Fig. 3A). These data suggested that both VSCC-SCs and SCC-SCs promoted the activation of 
A

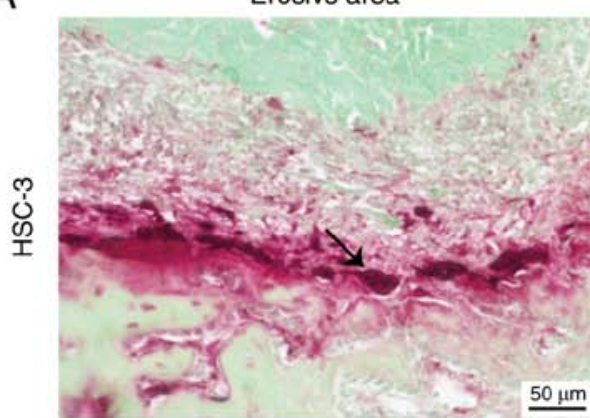

Mixed type
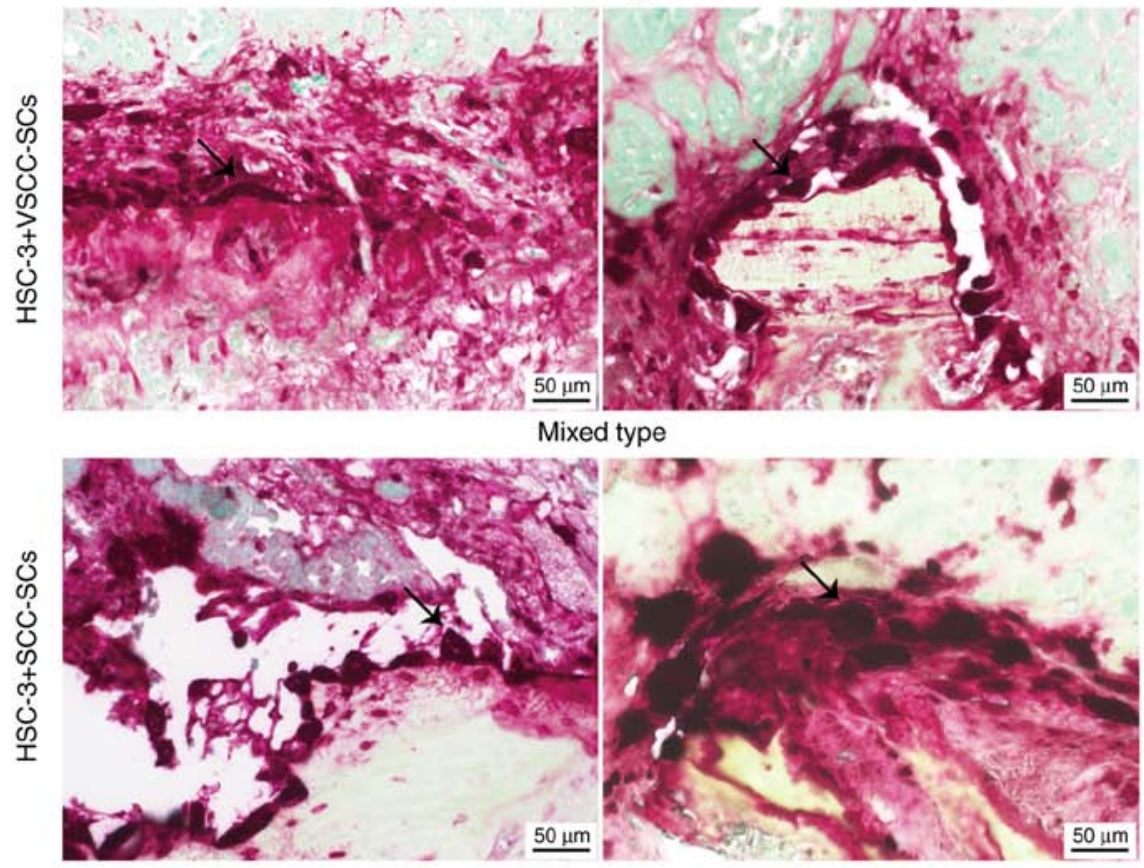

Mixed type

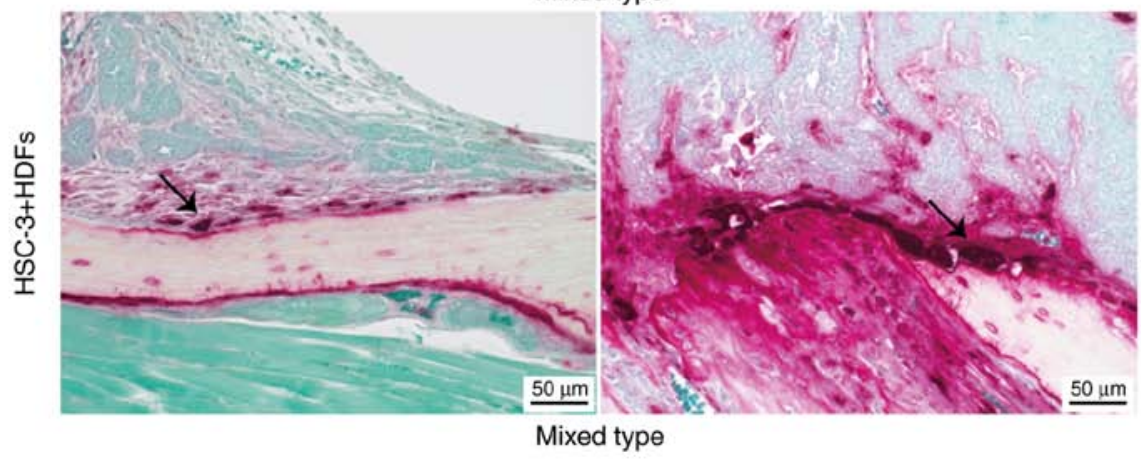

Infiltrative area

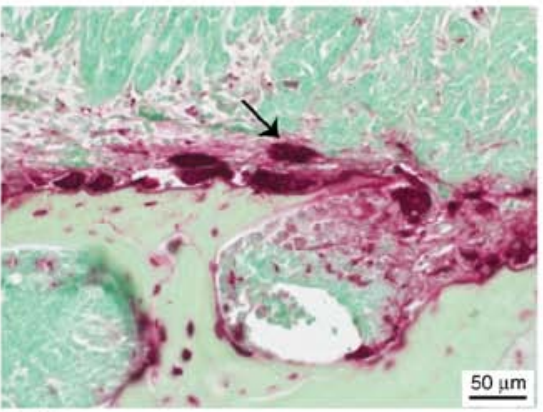

$\underline{50 \mu m}$
B

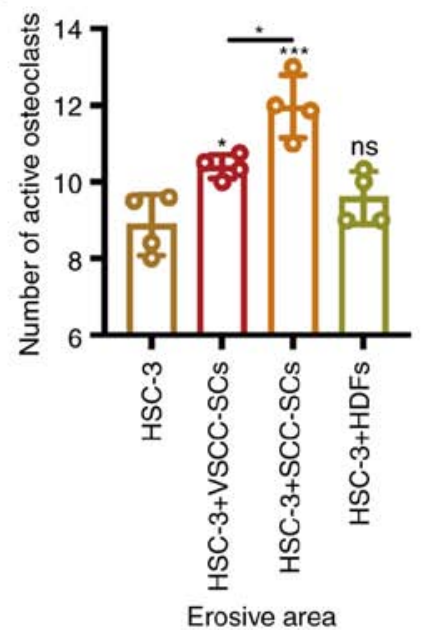

Figure 3. Effects of VSCC-SCs, SCC-SCs and HDFs on the activation of osteoclasts in the bone invasion areas of HSC-3 cells in vivo. (A) TRAP staining was used to determine the number of active multi-nucleated osteoclasts on bone surfaces. The arrows indicate the active multi-nucleated osteoclasts. (B) Semi-quantification of active multi-nucleated osteoclasts in the erosive part of different groups of HSC-3 cells. Data are shown as the mean \pm SD, $n=4$. Statistical analysis was performed by one-way ANOVA followed by Tukey's post hoc test. ${ }^{\text {ns }} \mathrm{P}>0.05,{ }^{*} \mathrm{P}<0.05,{ }^{* * *} \mathrm{P}<0.001 \mathrm{vs}$. HSC-3 or as indicated. HDFs, human dermal fibroblasts; SCC-SC, squamous cell carcinoma-associated stromal cells; VSCC-SC, verrucous squamous cell carcinoma-associated stromal cells.

osteoclasts in bone invasion regions of HSC-3 cells in vivo, and SCC-SCs had a more prominent effect than VSCC-SCs.

Both VSCC-SCs and SCC-SCs promote HSC-3 invasion in bone invasion regions in vivo by enhancing MMP9 and MT1-MMP expression. IHC was used to determine the expression levels of MMP9 and MT1-MMP in bone invasion areas, and to assess the effects of VSCC-SCs, SCC-SCs and HDFs on the invasion of HSC-3 cells (Fig. 4A and B). The IHC scores of
MMP9 and MT1-MMP in the HSC-3 + SCC-SCs group were slightly higher than those in the HSC-3 + VSCC-SCs group, and markedly higher than those in the HSC-3 and HSC-3 + HDFs groups, and these findings were significantly different, whereas there was little difference between the HSC-3 and HSC-3 + HDFs groups (Fig. 4C and D). These data suggested that both VSCC-SCs and SCC-SCs promoted the invasion of HSC-3 cells in bone invasion regions, and SCC-SCs had a more prominent effect than VSCC-SCs, whereas HDFs had a minimal effect. 
A

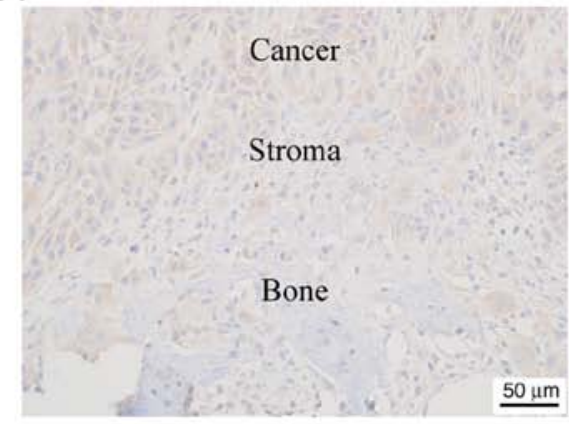

HSC-3+SCC-SCs

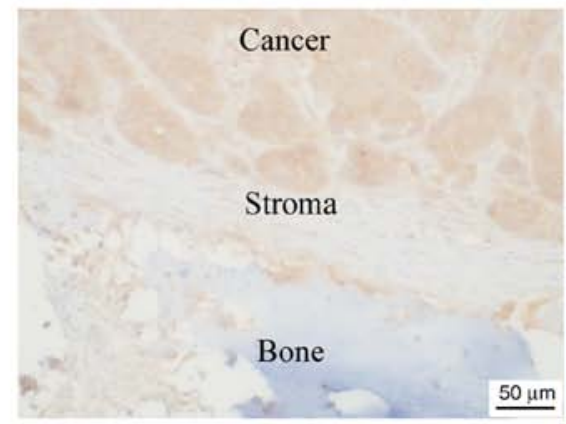

B

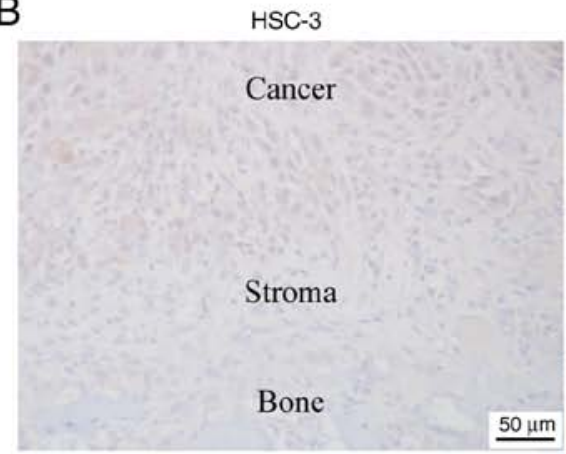

HSC-3+SCC-SCs

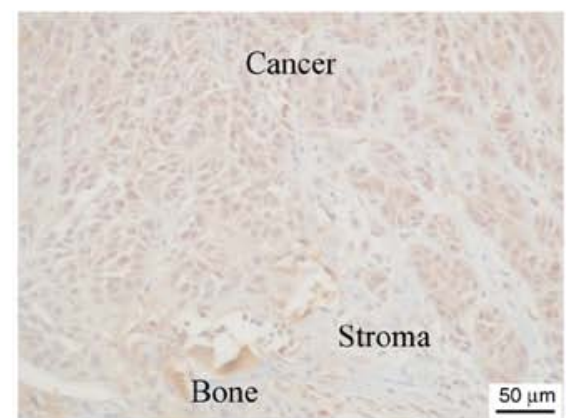

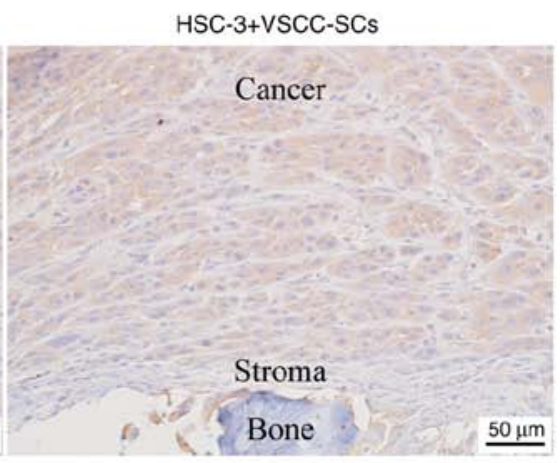

HSC-3+HDFs

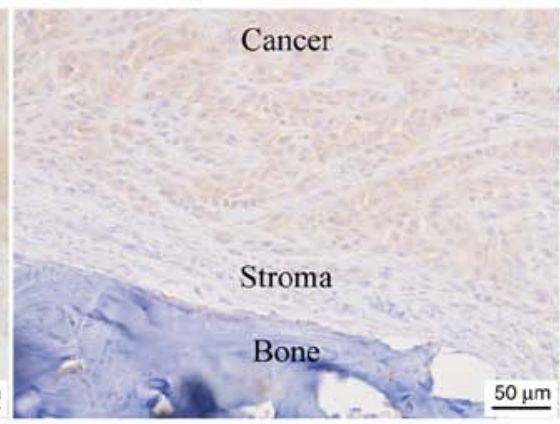

HSC-3+VSCC-SCs

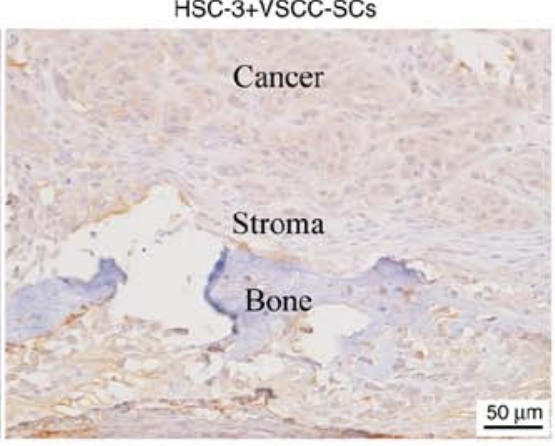

HSC-3+HDFs

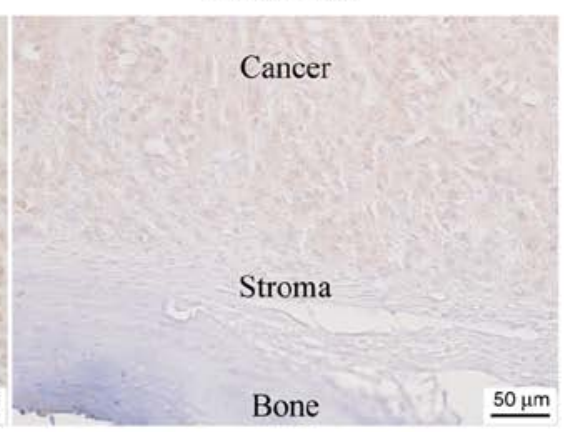

C

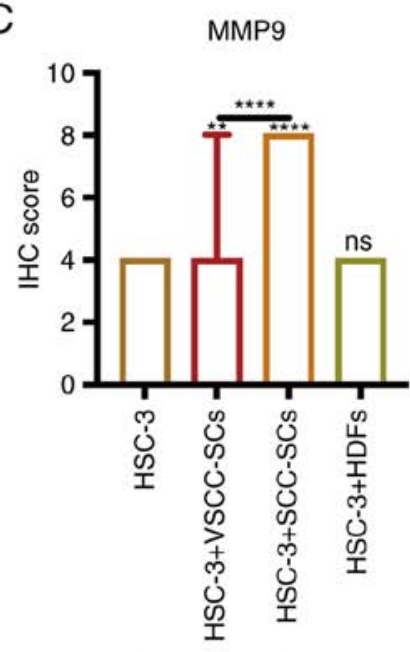

Erosive area (cancer)

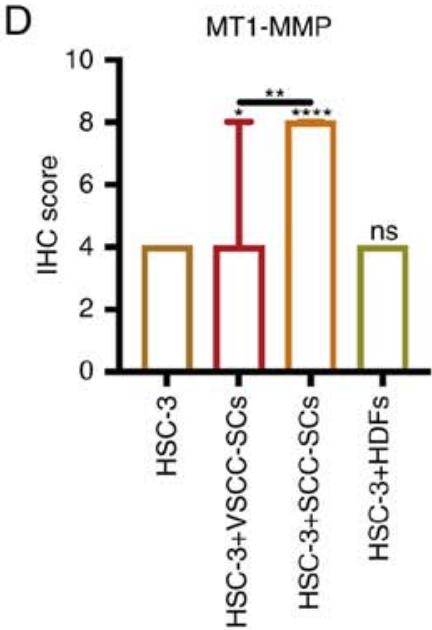

Erosive area (cancer)

Figure 4. Effects of VSCC-SCs, SCC-SCs and HDFs on the invasion of HSC-3 cells in bone invasion regions. IHC was used to determine the expression levels of (A) MMP9 and (B) MT1-MMP. Semi-quantification of (C) MMP9 and (D) MT1-MMP expression levels in bone invasion regions of HSC-3 cells. Data are shown as the median and IQR, $n=4$. Statistical analysis was performed by Kruskal-Wallis test followed by Dunn's test. ${ }^{\text {ns }} \mathrm{P}>0.05,{ }^{*} \mathrm{P}<0.05,{ }^{* *} \mathrm{P}<0.01,{ }^{* * * * * *} \mathrm{P}<0.0001$ vs. HSC-3 or as indicated. HDFs, human dermal fibroblasts; MMP, matrix metalloproteinase; IHC, immunohistochemistry; MT1-MMP, membrane-type 1 MMP; SCC-SC, squamous cell carcinoma-associated stromal cells; VSCC-SC, verrucous squamous cell carcinoma-associated stromal cells.

Both VSCC-SCs and SCC-SCs promote epithelialmesenchymal transition (EMT) of HSC-3 cells in bone invasion regions in vivo. IHC was used to detect the expression levels of Snail to determine the effect of VSCC-SCs, SCC-SCs and HDFs on the EMT of HSC-3 in bone invasion areas (Fig. 5A). The IHC score of Snail was highest in the HSC-3 + SCC-SCs group, closely followed by the HSC-3 + VSCC-SCs group. There was little difference between the HSC-3 and
HSC-3 + HDFs groups (Fig. 5B). These data indicated that both VSCC-SCs and SCC-SCs may enhance the EMT of HSC-3 cells in bone invasion regions, and SCC-SCs had a more prominent effect than VSCC-SCs, whereas HDFs had a minimal effect.

Both VSCC-SCs and SCC-SCs promote RANKL expression in cancer and stroma areas in the bone invasion region of 
A

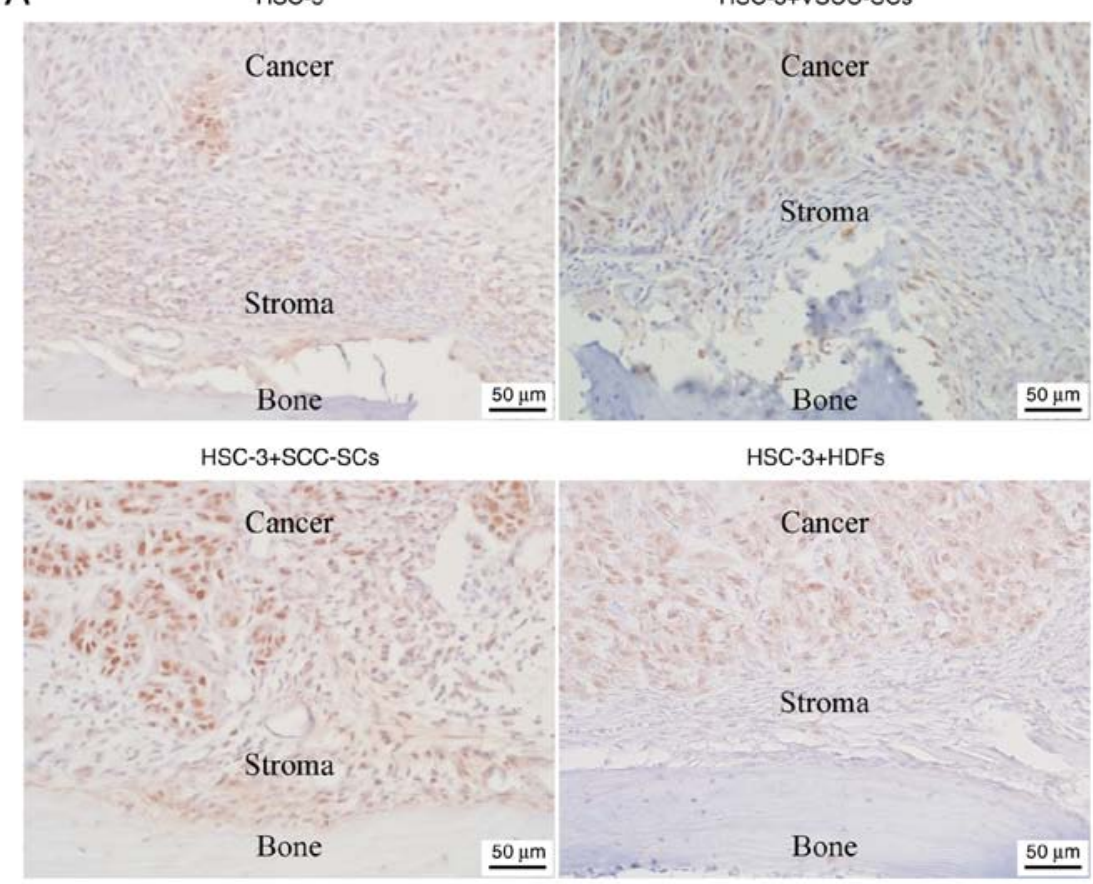

B

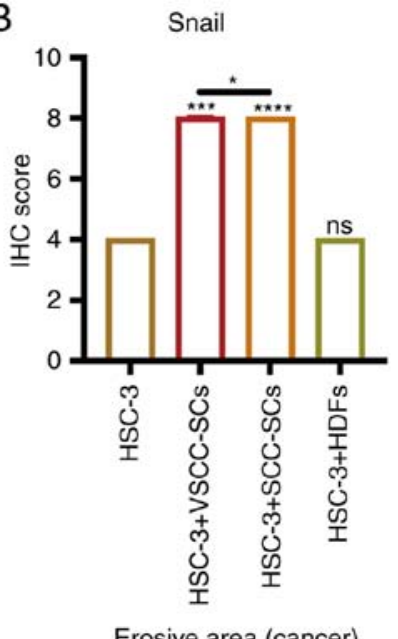

Erosive area (cancer)

Figure 5. Effects of VSCC-SCs, SCC-SCs and HDFs on the epithelial-mesenchymal transition of HSC-3 cells in bone invasion regions. (A) IHC was used to determine the expression levels of Snail. (B) Semi-quantification of Snail expression levels in the bone invasion regions of different groups of HSC-3 cells. Data are shown as the median and IQR, $\mathrm{n}=4$. Statistical analysis was performed by Kruskal-Wallis test followed by Dunn's test. ${ }^{\text {ns }} \mathrm{P}>0.05,{ }^{*} \mathrm{P}<0.05,{ }^{* * *} \mathrm{P}<0.001$, ${ }^{* * * * * *} \mathrm{P}<0.0001$ vs. HSC-3 or as indicated. HDFs, human dermal fibroblasts; IHC, immunohistochemistry; SCC-SC, squamous cell carcinoma-associated stromal cells; VSCC-SC, verrucous squamous cell carcinoma-associated stromal cells.

HSC-3 cells in vivo. IHC was used to determine the expression levels of RANKL in cancer and stroma areas to determine the effects of VSCC-SCs, SCC-SCs and HDFs on the activation of osteoclasts in the bone invasion areas of HSC-3 cells (Fig. 6A). Semi-quantification of IHC scores was only conducted in the erosive area to present the protein expression levels in cancer regions. The protein expression levels in stroma regions were only compared by intensity rather than by semi-quantification of IHC score. In stroma areas, the intensities of RANKL in HSC-3 + VSCC-SCs and HSC-3 + SCC-SCs groups were slightly higher than those in the HSC-3 and HSC-3 + HDFs groups (Fig. 6A). In cancer areas, the IHC score of RANKL was highest in the HSC-3 + SCC-SCs group, followed by the HSC-3 + VSCC-SCs group; there was little difference between the HSC-3 and HSC-3 + HDFs groups (Fig. 6B). These data demonstrated that both VSCC-SCs and SCC-SCs promoted RANKL expression in cancer and stroma areas in the bone invasion regions of HSC-3 cells in vivo, and that SCC-SCs had a stronger effect than VSCC-SCs, whereas HDFs had a minimal effect.

Both VSCC-SCs and SCC-SCs promote PTHrP expression in cancer and stroma areas in bone invasion regions of HSC-3 cells in vivo. Given that RANKL expression is regulated by PTHrP (12), IHC was used to determine the expression levels of PTHrP in cancer and stroma areas in bone invasion regions of HSC-3 cells (Fig. 7A). Semi-quantification of IHC scores was only conducted in the erosive area to present the protein expression levels in cancer regions. The protein expression levels in stroma regions were only compared by intensity rather than by semi-quantification of IHC score. In stroma areas, the intensities of PTHrP in the HSC-3 + SCC-SCs and HSC-3 + VSCC-SCs groups were markedly higher than those in the HSC-3 and HSC-3 + HDFs groups (Fig. 7A). In cancer areas, the IHC score of PTHrP in the HSC-3 + SCC-SCs was slightly higher than that in the HSC-3 + VSCC-SCs group, but was markedly higher than that in the HSC-3 + HDFs and HSC-3 groups; these findings were significantly different. There was little difference between the HSC-3 and HSC-3 + HDFs groups (Fig. 7B). These data suggested that both VSCC-SCs and SCC-SCs promoted PTHrP expression in cancer and stroma areas in the bone invasion regions of HSC-3 cells in vivo, and SCC-SCs had a greater effect than VSCC-SCs, whereas HDFs exerted a minimal effect.

ILIB, ICAM1, FOS, CXCL12, INS and NGF may underlie the differential effects of VSCC-SCs and SCC-SCs on bone invasion in OSCC. Microarray data were used to analyze the upregulated DEGs in SCC-SCs compared with in VSCC-SCs. The upregulated DEGs that could interact with MMP9, MMP14, Snail, RANKL and PTHrP were identified by a PPI network (Fig. 8A). The hub genes in these upregulated DEGs were also identified by PPI. The results revealed that IL6, ICAM1, CXCL12, IL1B, CDH1, CDC42, FOS, TLR4, NGF and INS were hub genes (Fig. 8B). The hub genes that were differentially expressed in VSCC-SCs and SCC-SCs were then analyzed by heatmap, which indicated that IL1B, ICAM1, FOS, CXCL12, INS and NGF were differentially expressed in SCC-SCs compared with in VSCC-SCs (Fig. 8C). In addition, IL1B, ICAM1, FOS, CXCL12 and INS can interact with MMP9, MMP14, Snail, RANKL and PTHrP, whereas NGF can only interact with MMP9, MMP14 and Snail, according 
A
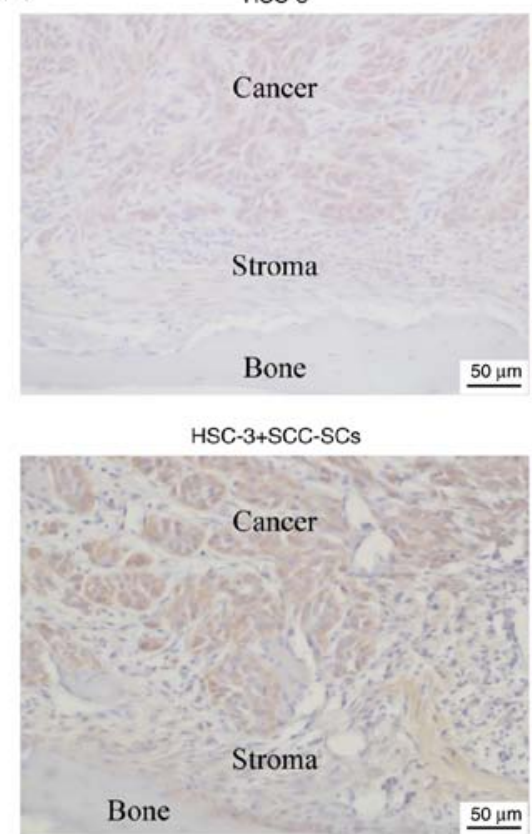

HSC-3+VSCC-SCs

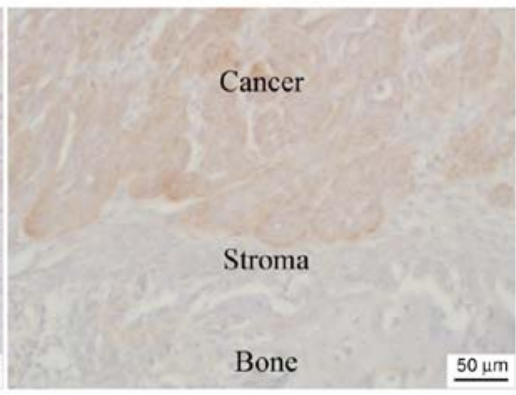

HSC-3+HDFs

Cancer

Stroma

B

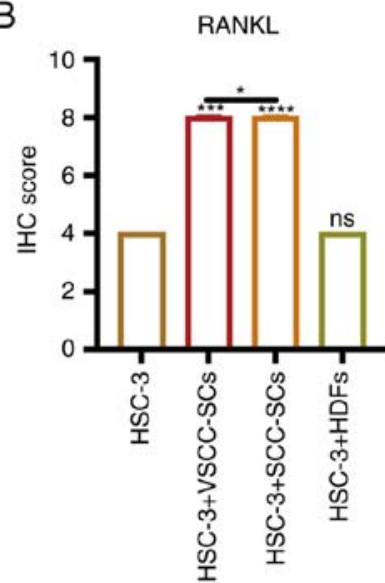

Erosive area (cancer)

Figure 6. Effects of VSCC-SCs, SCC-SCs and HDFs on RANKL expression in cancer and stroma areas in bone invasion regions of HSC-3 cells in vivo. (A) IHC was used to determine RANKL expression in cancer and stroma areas. (B) Semi-quantification of RANKL expression in different groups of HSC-3 cells. Data are shown as the median and IQR, $\mathrm{n}=4$. Statistical analysis was performed by Kruskal-Wallis test followed by Dunn's test. ${ }^{\mathrm{n}} \mathrm{P}>0.05$, ${ }^{*} \mathrm{P}<0.05$, ${ }^{* * * *} \mathrm{P}<0.001,{ }^{* * * *} \mathrm{P}<0.0001$ vs. HSC-3 or as indicated. HDFs, human dermal fibroblasts; IHC, immunohistochemistry; RANKL, receptor activator of NF- $\mathrm{KB}$ ligand; SCC-SC, squamous cell carcinoma-associated stromal cells; VSCC-SC, verrucous squamous cell carcinoma-associated stromal cells.

A
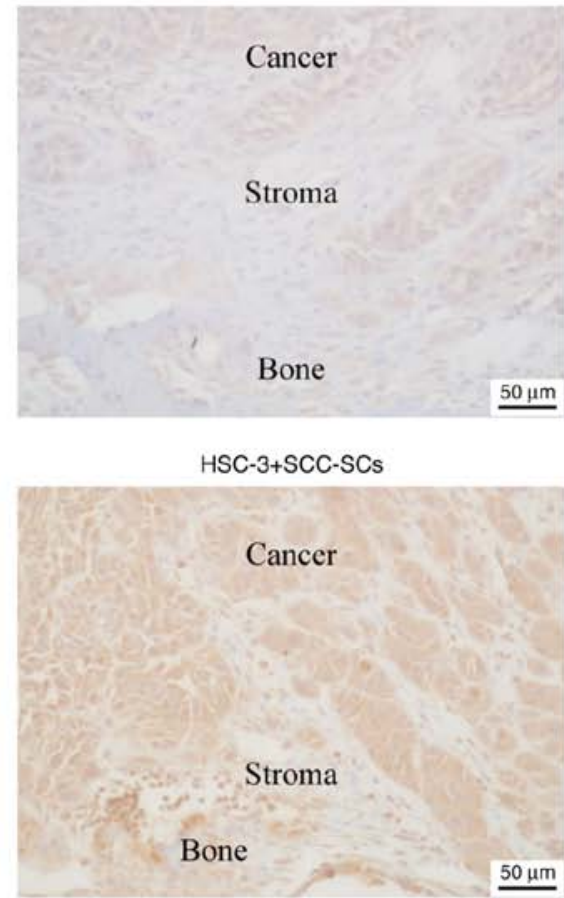

HSC-3+VSCC-SCS

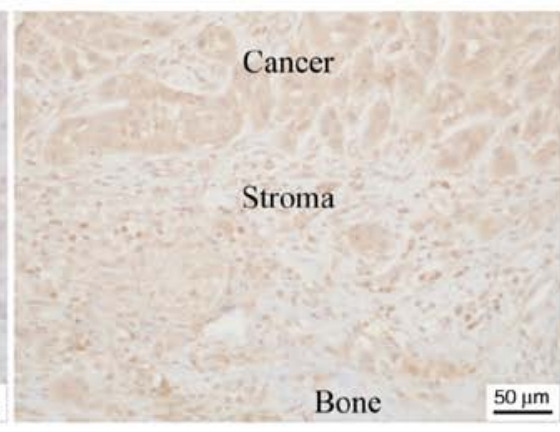

HSC-3+HDFs

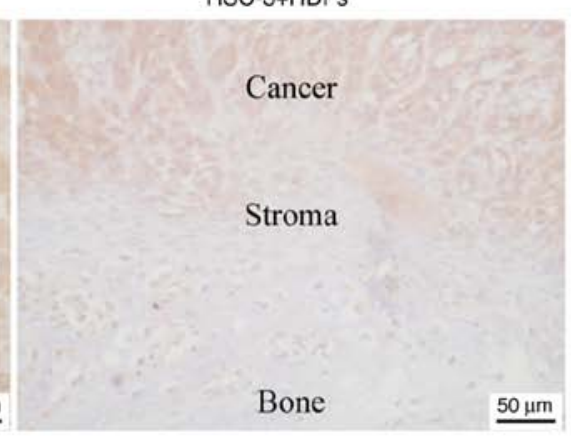

B

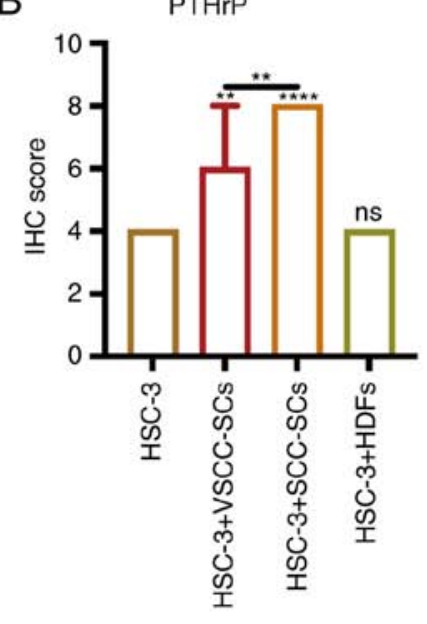

Erosive area (cancer)

Figure 7. Effects of VSCC-SCs, SCC-SCs and HDFs on PTHrP in cancer and stroma areas in the bone invasion regions of HSC-3 cells in vivo. (A) IHC was used to determine PTHrP expression in different groups of HSC-3 cells. (B) Semi-quantification of PTHrP expression in different groups of HSC-3 cells. Data

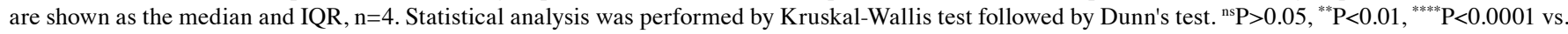
HSC-3 or as indicated. HDFs, human dermal fibroblasts; IHC, immunohistochemistry; PTHrP, parathyroid hormone-related peptide; SCC-SC, squamous cell carcinoma-associated stromal cells; VSCC-SC, verrucous squamous cell carcinoma-associated stromal cells.

to the results of PPI and the Venn diagram (Fig. 8D). These data suggested that IL1B, ICAM1, FOS, CXCL12, INS and
NGF may underlie the differential effects of VSCC-SCs and SCC-SCs on bone invasion of OSCC. 

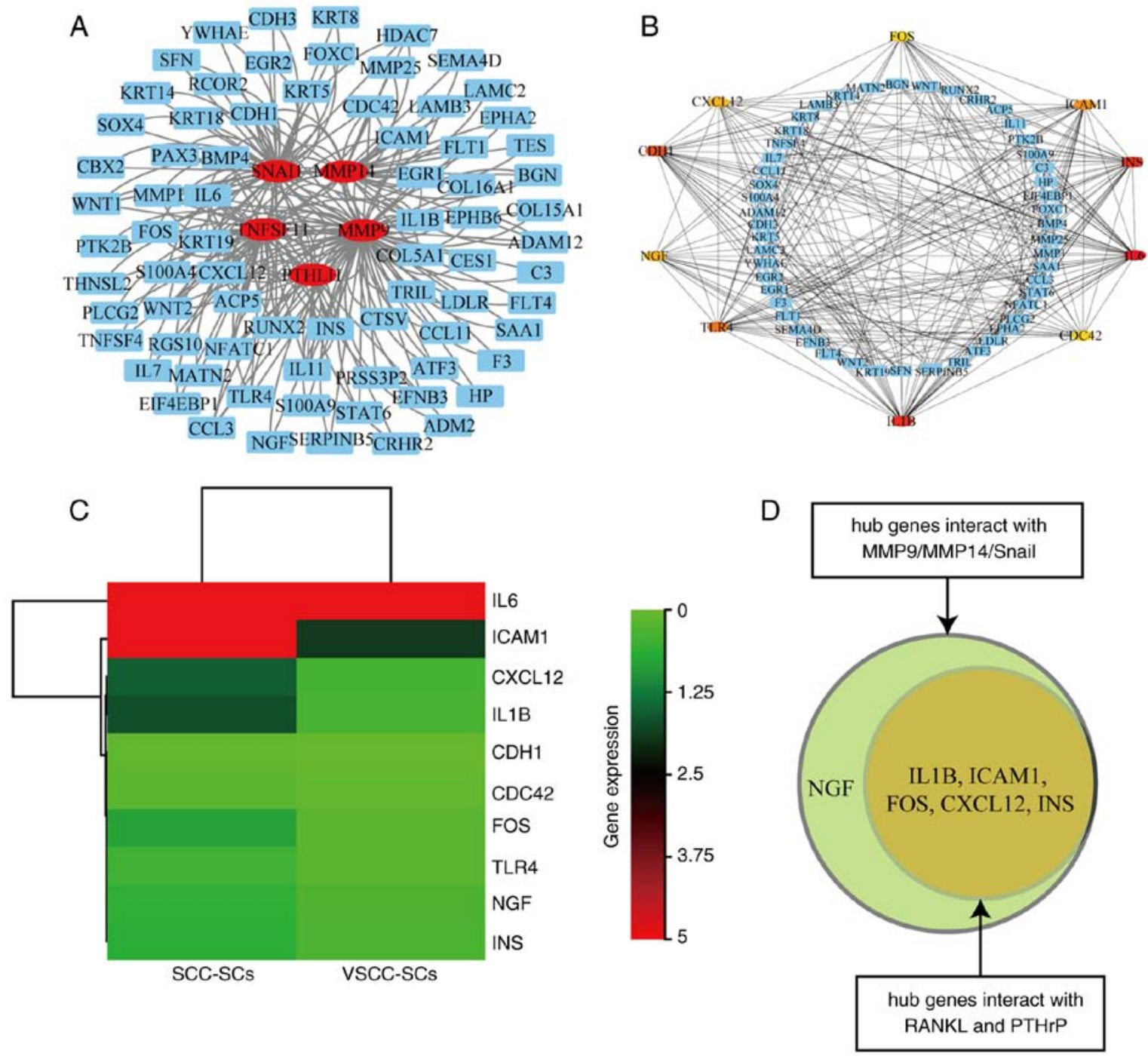

Figure 8. Identification of potential genes that underlie the differential effects of VSCC-SCs and SCC-SCs on bone invasion in OSCC. (A) PPI analysis identified the upregulated DEGs in SCC-SCs that could interact with MMP9, MMP14, Snail, RANKL and PTHrP. (B) PPI analysis identified the hub genes in the upregulated DEGs in SCC-SCs that could interact with MMP9, MMP14, Snail, RANKL and PTHrP. (C) Hub genes differentially expressed in VSCC-SCs and SCC-SCs were examined using a heatmap. (D) Relevant genes that the differentially expressed hub genes could interact with are presented using a Venn plot. DEGs, differentially expressed genes; MMP, matrix metalloproteinase; OSCC, oral squamous cell carcinoma; PPI, protein-protein interaction; PTHrP, parathyroid hormone-related peptide; RANKL, receptor activator of NF- $\mathrm{kB}$ ligand; SCC-SC, squamous cell carcinoma-associated stromal cells; VSCC-SC, verrucous squamous cell carcinoma-associated stromal cells.

\section{Discussion}

Invasion of OSCC into the nearby maxilla and mandible can promote progression of the tumor stage. Bone invasion is diagnosed by CT and MRI, and the treatment method is bone resection (28-30). There are three types of OSCC bone invasion: Erosive, infiltrative and mixed (26,27). Our research group previously reported that both VSCC-SCs and $\mathrm{SCC}-\mathrm{SC}$ could promote the bone invasion of $\mathrm{HSC}-2$ and HSC-3 cells. Notably, SCC-SCs were revealed to have a better promoting effect than VSCC-SCs on the bone invasion of HSC-2 and HSC-3 cells, whereas HDFs exerted a minimal effect; however, these two studies did not assess the type of bone invasion and the relevant regulatory mechanisms $(21,22)$. The present study mainly focused on the type of invasion and the potential regulatory mechanism. The results revealed that the $\mathrm{HSC}-3$, HSC-3 + SCC-SCs, HSC-3 + VSCC-SCs and HSC-3 + HDFs groups exhibited mixed-type bone invasion. In addition, both SCC-SCs and VSCC-SCs promoted the bone invasion of HSC-3 cells in vitro and in vivo, and SCC-SCs had a more prominent effect than VSCC-SCs, whereas HDFs had a minimal effect. A previous study indicated that there are numerous giant osteoclasts with different shapes on the bone surfaces in bone invasion areas of OSCC (31). In the present study, the giant osteoclasts in the HSC-3 + SCC-SCs group were slightly larger than those in the HSC-3 + VSCC-SCs and HSC-3 groups, but were markedly larger than those in the HSC-3 + HDFs group, in vitro and in vivo. Therefore, the size of osteoclasts could influence bone resorption in OSCC. In addition, the HSC-3 + SCC-SCs group contained triangle- and round-shaped osteoclasts, whereas the HSC-3 + VSCC-SCs group contained triangle-, ellipse- and round-shaped osteoclasts, and there were ellipse- and round-shaped osteoclasts in the HSC-3 and HSC-3 + HDFs groups. With regard to their bone invasion degree, the present findings indicated that the 
triangle-shaped osteoclasts have the best bone resorption ability, followed by ellipse-shaped osteoclasts, whereas the round-shaped osteoclasts exert a minimal effect on the bone resorption. Triangle-shaped osteoclasts are activated multinucleated osteoclasts that are formed by the fusion of several osteoclasts; therefore, the triangle-shaped osteoclasts have the best bone invasion ability. Ellipse-shaped osteoclasts are a kind of spindle-shaped osteoclast, which are more active than normal osteoclasts but are not formed by the fusion of several osteoclasts; therefore, the bone invasion ability of ellipse-shaped osteoclasts is lower than that of triangle-shaped osteoclasts. Round-shaped osteoclasts are similar to the shape of normal osteoclasts and have the lowest bone invasion ability. All of these findings have been indirectly confirmed by a previous study (30). Therefore, the shape of the osteoclasts may be associated with bone resorption. In conclusion, both SCC-SCs and VSCC-SCs influenced the bone invasion of OSCC by regulating the number, size and shape of giant osteoclasts on bone surfaces.

The bone invasion of OSCC is divided into three phases: Initial phase, bone resorption phase and final phase (32). In the initial phase, MMPs serve a significant role in the crosstalk between cancer cells and bone-relevant cells by promoting cancer cells to enter into soft tissues or bone marrow spaces $(33,34)$. Furthermore, cancer cells can migrate into the TME to invade the bone tissues via EMT (35). P130cas is one factor that can promote the bone invasion of OSCC by regulating EMT (36). Furthermore, TGF- $\beta 1$ can promote EMT and subsequently promote the OSCC bone invasion by enhancing the activation of osteoclasts (37). Given that both cancer and stroma have an effect on the bone invasion of OSCC, semi-quantification of IHC scores was only conducted in the erosive area to present the relevant protein expression levels in cancer regions. The relevant protein expression levels in stroma regions were only compared by intensity rather than by semi-quantification of IHC score. In the present study, SCC-SCs and VSCC-SCs promoted MMP9 and MT1-MMP expression, and the EMT of HSC-3 cells in bone invasion areas, with SCC-SCs having a more prominent effect. Therefore, VSCC-SCs and especially SCC-SCs may promote the initial phase of bone invasion of OSCC, whereas HDFs have a minimal effect.

The second phase of bone invasion of OSCC is bone resorption mediated by osteoclasts (32). Osteoclast formation is regulated by colony-stimulating factor 1 and RANKL (38). OSCC bone invasion could be induced by the expression of RANKL, which promotes osteoclast formation in the TME (39). The inhibition of RANK and RANKL has been shown to suppress the bone invasion of OSCC by inhibiting osteoclast formation $(40,41)$. A previous study also indicated that RANKL expression is regulated by PTHrP (42). Overexpression of PTHrP in OSCC has been reported to promote RANKL expression, which in turn may regulate the formation of osteoclasts in the TME $(12,43)$. In the present study, SCC-SCs and VSCC-SCs promoted the expression levels of RANKL and PTHrP in cancer and stroma areas of bone invasion regions of HSC-3 cells in vivo, with SCC-SCs having a more prominent effect. Therefore, VSCC-SCs and especially SCC-SCs may promote the bone resorption phase of bone invasion of OSCC, whereas HDFs have little effect. The present study only revealed that VSCC-SCs and SCC-SCs could promote bone invasion by enhancing the expression levels of RANKL and PTHrP; however, whether the VSCC-SCs and SCC-SCs regulate bone invasion of OSCC by PTHrP/RANKL pathways needs to be further investigated by using the RANKL and PTHrP inhibitor. In addition, due to the direct co-culture method used in the present study, protein or RNA could not be extracted from a certain cell or tissue type independently. Therefore, IHC was performed to determine the expression levels of the relevant proteins, which is not as accurate as western blotting and reverse transcription-quantitative PCR.

The findings of microarray and bioinformatics analysis suggested that IL1B, ICAM1, FOS, CXCL12, INS and NGF may underlie the differential effects of VSCC-SCs and SCC-SCs on bone invasion in OSCC. A previous study indicated that IL1B is closely associated with the EMT of human gastric adenocarcinoma cells, which also promotes the formation of multinucleated osteoclasts by enhancing RANKL-dependent adseverin expression $(44,45)$. ICAM1 has been reported to be closely associated with MMP9 and MMP14 expression in breast cancer, and soluble ICAM1 and RANKL have synergistic effects on the activation of osteoclasts (46-49). PTHrP can also regulate ICAM1 expression by influencing TGF- $\beta$ (50). The Fos proto-oncogene, FOS, has also been reported to be closely associated with the expression of MMP9 in breast cancer and in the EMT process of hepatocellular carcinoma $(51,52)$, and a recent study indicated that umbelliferone may inhibit RANKL-induced osteoclast formation by suppressing the Akt-c-Fos-NFATc1 signaling pathway (53). In addition, PTHrP can promote FOS expression in cementoblasts (54). INS has been poorly investigated in the bone invasion of OSCC. CCXCL12 and CXCR4 have been shown to serve a critical role in the invasion of OSCC and EMT of breast cancer $(55,56)$, and CXCL12 may also enhance RANKL- and TNF- $\alpha$-induced osteoclastogenesis and bone resorption (57). Therefore, CXCL12 may regulate the initial and bone resorption phases of bone invasion of OSCC. A recent study suggested that NGF promotes MMP9 expression indirectly in colorectal cancer metastasis (58), and the NGF-TrkA axis enhances EMT and EGFR inhibitor resistance by activating the STAT3 signaling pathway in head and neck squamous cell carcinoma (59). In addition, NGF has been demonstrated to be closely associated with bone resorption in periodontitis (60). It is clear that these aforementioned factors have the potential to regulate the bone invasion of OSCC. However, all of these potential genes were only analyzed by microarray, which represents their mRNA expression levels in the stromal cells. The protein expression levels of these potential genes in stromal cells need to be further confirmed by western blotting.

In conclusion, both SCC-SCs and VSCC-SCs promoted the bone invasion of OSCC by regulating the initial and bone resorption phases, and SCC-SCs had a more prominent effect. IL1B, ICAM1, FOS, CXCL12, INS and NGF may underlie the differential effects of VSCC-SCs and SCC-SCs on the bone invasion of OSCC. These findings illuminate the potential regulatory mechanisms of bone invasion in OSCC, which could contribute to the treatment and prognosis of patients with OSCC. 


\section{Acknowledgements}

Not applicable.

\section{Funding}

This work was supported by the Japan Society for Promotion of Science (JSPS) KAKENHI Grants-in-Aid for Scientific Research (grant nos. JP21K17089, JP19K19159, JP20K10178, JP20H03888 and JP21K10043).

\section{Availability of data and materials}

The datasets used and/or analyzed during the current study are available from the corresponding author on reasonable request. The microarray data generated in the present study may be found in the Gene Expression Omnibus under accession number GSE164374 or at the following URL: https://www. ncbi.nlm.nih.gov/geo/query/acc.cgi?\&acc=GSE164374.

\section{Authors' contributions}

QS designed the outline of the study. QS, KT, HK, MWO, YI, $\mathrm{SS}, \mathrm{SF}, \mathrm{KN}$ and $\mathrm{HN}$ conducted experiments and data analysis. QS and KT were involved in the preparation of the manuscript. QS wrote the manuscript. KN and HN supervised the study, and contributed to data interpretation and manuscript revision. KT and HN confirmed the authenticity of all raw data. All authors have read and approve the final manuscript.

\section{Ethics approval and consent to participate}

The present study was approved by the Ethics Committee of Okayama University (project identification code: 1703-042-001). Written informed consent was obtained from all patients. All animal experiments were conducted according to the relevant guidelines and regulations approved by the institutional committees at Okayama University (approval no. OKU-2017406).

\section{Patient consent for publication}

Not applicable.

\section{Competing interests}

The authors declare that they have no competing interests.

\section{References}

1. Warnakulasuriya S: Global epidemiology of oral and oropharyngeal cancer. Oral Oncol 45: 309-316, 2009.

2. Sacco AG and Cohen EE: Current treatment options for recurrent or metastatic head and neck squamous cell carcinoma. J Clin Oncol 33: 3305-3313, 2015.

3. Nomura T, Shibahara T, Cui NH and Noma H: Patterns of mandibular invasion by gingival squamous cell carcinoma. J Oral Maxillofac Surg 63: 1489-1493, 2005.

4. Chen YL, Kuo SW, Fang KH and Hao SP: Prognostic impact of marginal mandibulectomy in the presence of superficial bone invasion and the nononcologic outcome. Head Neck 33: 708-713, 2011.

5. Teitelbaum SL and Ross FP: Genetic regulation of osteoclast development and function. Nat Rev Genet 4: 638-649, 2003.
6. Takayanagi H: Osteoimmunology: Shared mechanisms and crosstalk between the immune and bone systems. Nat Rev Immunol 7: 292-304, 2007.

7. Teitelbaum SL: Osteoclasts: What do they do and how do they do it? Am J Pathol 170: 427-435, 2007.

8. Okamoto M, Hiura K, Ohe G, Ohba Y, Terai K, Oshikawa T, Furuichi S, Nishikawa H, Moriyama K, Yoshida H and Sato M: Mechanism for bone invasion of oral cancer cells mediated by interleukin-6 in vitro and in vivo. Cancer 89: 1966-1975, 2000.

9. Tada T, Jimi E, Okamoto M, Ozeki S and Okabe K: Oral squamous cell carcinoma cells induce osteoclast differentiation by suppression of osteoprotegerin expression in osteoblasts. Int J Cancer 116: 253-262, 2005.

10. Ono K, Akatsu T, Kugai N, Pilbeam CC and Raisz LG: The effect of deletion of cyclooxygenase-2, prostaglandin receptor EP2, or EP4 in bone marrow cells on osteoclasts induced by mouse mammary cancer cell lines. Bone 33: 798-804, 2003.

11. Jimi E, Furuta H, Matsuo K, Tominaga K, Takahashi T and Nakanishi O: The cellular and molecular mechanisms of bone invasion by oral squamous cell carcinoma. Oral Dis 17: 462-468, 2011.

12. Kayamori K, Sakamoto K, Nakashima T, Takayanagi H, Morita K, Omura K, Nguyen ST, Miki Y, Iimura T, Himeno A, et al: Roles of interleukin-6 and parathyroid hormone-related peptide in osteoclast formation associated with oral cancers: Significance of interleukin- 6 synthesized by stromal cells in response to cancer cells. Am J Pathol 176: 968-980, 2010.

13. Valkenburg KC, de Groot AE and Pienta KJ: Targeting the tumour stroma to improve cancer therapy. Nat Rev Clin Oncol 15: 366-381, 2018.

14. Quail DF and Joyce JA: Microenvironmental regulation of tumor progression and metastasis. Nat Med 19: 1423-1437, 2013.

15. Wei LY, Lee JJ, Yeh CY, Yang CJ, Kok SH, Ko JY, Tsai FC and Chia JS: Reciprocal activation of cancer-associated fibroblasts and oral squamous carcinoma cells through CXCL1. Oral Oncol 88: 115-123, 2019.

16. An YZ, Cho E, Ling $J$ and Zhang X: The Axin2-snail axis promotes bone invasion by activating cancer-associated fibroblasts in oral squamous cell carcinoma. BMC Cancer 20: 987, 2020.

17. Elmusrati AA, Pilborough AE, Khurram SA and Lambert DW: Cancer-associated fibroblasts promote bone invasion in oral squamous cell carcinoma. Br J Cancer 117: 867-875, 2017.

18. Patel SG and Shah JP: TNM staging of cancers of the head and neck: Striving for uniformity among diversity. CA Cancer J Clin 55: 242-258, 2005.

19. Spiro RH, Guillamondegui O Jr, Paulino AF and Huvos AG: Pattern of invasion and margin assessment in patients with oral tongue cancer. Head Neck 21: 408-413, 1999.

20. Hussein MR and Cullen K: Molecular biomarkers in HNSCC: prognostic and therapeutic implications. Expert Rev Anticancer Ther 1: 116-124, 2001.

21. Takabatake K, Kawai H, Omori H, Qiusheng S, Oo MW, Sukegawa S, Nakano K, Tsujigiwa $H$ and Nagatsuka H: Impact of the stroma on the biological characteristics of the parenchyma in oral squamous cell carcinoma. Int J Mol Sci 21: 7714, 2020.

22. Shan Q, Takabatake K, Omori H, Kawai H, Oo MW, Nakano K, Ibaragi S, Sasaki A and Nagatsuka H: Stromal cells in the tumor microenvironment promote the progression of oral squamous cell carcinoma. Int J Oncol 59: 72, 2021.

23. Tohyama R, Kayamori K, Sato K, Hamagaki M, Sakamoto K, Yasuda $\mathrm{H}$ and Yamaguchi A: Establishment of a xenograft model to explore the mechanism of bone destruction by human oral cancers and its application to analysis of role of RANKL. J Oral Pathol Med 45: 356-364, 2016.

24. Flecknell PA: Laboratory Animal Anesthesia. 3rd edition. Academic Press, San Diego, CA, 2009.

25. Chuang FH, Hsue SS, Wu CW and Chen YK: Immunohistochemical expression of RANKL, RANK, and OPG in human oral squamous cell carcinoma. J Oral Pathol Med 38: 753-758, 2009.

26. Shaw RJ, Brown JS, Woolgar JA, Lowe D, Rogers SN and Vaughan ED: The influence of the pattern of mandibular invasion on recurrence and survival in oral squamous cell carcinoma. Head Neck 26: 861-869, 2004.

27. Brown JS, Lowe D, Kalavrezos N, D'Souza J, Magennis P and Woolgar J: Patterns of invasion and routes of tumor entry into the mandible by oral squamous cell carcinoma. Head Neck 24: 370-383, 2002.

28. Uribe S, Rojas LA and Rosas CF: Accuracy of imaging methods for detection of bone tissue invasion in patients with oral squamous cell carcinoma. Dentomaxillofac Radiol 42: 20120346, 2013. 
29. Gibo T, Yamada SI, Kawamoto M, Uehara T and Kurita $\mathrm{H}$ : Immunohistochemical investigation of predictive biomarkers for mandibular bone invasion in oral squamous cell carcinoma. Pathol Oncol Res 26: 2381-2389, 2020.

30. Patel RS, Dirven R, Clark JR, Swinson BD, Gao K and O'Brien CJ: The prognostic impact of extent of bone invasion and extent of bone resection in oral carcinoma. Laryngoscope 118: 780-785, 2008.

31. Quan J, Hou Y, Long W, Ye S and Wang Z: Characterization of different osteoclast phenotypes in the progression of bone invasion by oral squamous cell carcinoma. Oncol Rep 39: 1043-1051, 2018.

32. Quan J, Johnson NW, Zhou G, Parsons PG, Boyle GM and Gao J: Potential molecular targets for inhibiting bone invasion by oral squamous cell carcinoma: A review of mechanisms. Cancer Metastasis Rev 31: 209-219, 2012.

33. Quan J, Zhou C, Johnson NW, Francis G, Dahlstrom JE and Gao J: Molecular pathways involved in crosstalk between cancer cells, osteoblasts and osteoclasts in the invasion of bone by oral squamous cell carcinoma. Pathology 44: 221-227, 2012.

34. Woodward JK, Holen I, Coleman RE and Buttle DJ: The roles of proteolytic enzymes in the development of tumour-induced bone disease in breast and prostate cancer. Bone 41: 912-927, 2007.

35. van der Pluijm G: Epithelial plasticity, cancer stem cells and bone metastasis formation. Bone 48: 37-43, 2011.

36. Yaginuma T, Gao J, Nagata K, Muroya R, Fei H, Nagano $H$, Chishaki S, Matsubara T, Kokabu S, Matsuo K, et al: p130Cas induces bone invasion by oral squamous cell carcinoma by regulating tumor epithelial-mesenchymal transition and cell proliferation. Carcinogenesis 41: 1038-1048, 2020.

37. Quan J,Elhousiny M, Johnson NW and Gao J: Transforming growth factor- $\beta 1$ treatment of oral cancer induces epithelial-mesenchymal transition and promotes bone invasion via enhanced activity of osteoclasts. Clin Exp Metastasis 30: 659-670, 2013.

38. Raggatt LJ and Partridge NC: Cellular and molecular mechanisms of bone remodeling. J Biol Chem 285: 25103-25108, 2010

39. Sambandam Y, Ethiraj P, Hathaway-Schrader JD, Novince CM, Panneerselvam E, Sundaram K and Reddy SV: Autoregulation of RANK ligand in oral squamous cell carcinoma tumor cells. J Cell Physiol 233: 6125-6134, 2018.

40. Shin M, Matsuo K, Tada T, Fukushima H, Furuta H, Ozeki S, Kadowaki T, Yamamoto K, Okamoto $M$ and Jimi E: The inhibition of RANKL/RANK signaling by osteoprotegerin suppresses bone invasion by oral squamous cell carcinoma cells. Carcinogenesis 32: 1634-1640, 2011

41. Zhang X, Junior CR, Liu M, Li F, D'Silva NJ and Kirkwood KL: Oral squamous carcinoma cells secrete RANKL directly supporting osteolytic bone loss. Oral Oncol 49: 119-128, 2013.

42. Takayama Y, Mori T, Nomura T, Shibahara T and Sakamoto $M$ : Parathyroid-related protein plays a critical role in bone invasion by oral squamous cell carcinoma. Int J Oncol 36: 1387-1394, 2010

43. Martin CK, Dirksen WP, Shu ST, Werbeck JL, Thudi NK, Yamaguchi M, Wolfe TD, Heller KN and Rosol TJ: Characterization of bone resorption in novel in vitro and in vivo models of oral squamous cell carcinoma. Oral Oncol 48: 491-499, 2012.

44. Wang X, Wang B, Xie J, Hou D, Zhang H and Huang H: Melatonin inhibits epithelial-to-mesenchymal transition in gastric cancer cells via attenuation of IL-1 $\beta / \mathrm{NF}-\kappa \mathrm{B} / \mathrm{MMP} / \mathrm{MMP} 9$ signaling. Int J Mol Med 42: 2221-2228, 2018.

45. Wang Y, Galli M, Shade Silver A, Lee W, Song Y, Mei Y, Bachus C, Glogauer $M$ and McCulloch CA: IL1 $\beta$ and TNFo promote RANKL-dependent adseverin expression and osteoclastogenesis. J Cell Sci 131: 213967, 2018.

46. Chung TW, Choi H, Lee JM, Ha SH, Kwak CH, Abekura F, Park JY, Chang YC, Ha KT, Cho SH, et al: Oldenlandia diffusa suppresses metastatic potential through inhibiting matrix metalloproteinase-9 and intercellular adhesion molecule-1 expression via p38 and ERK1/2 MAPK pathways and induces apoptosis in human breast cancer MCF-7 cells. J Ethnopharmacol 195: 309-317, 2017.
47. Di D, Chen L, Wang L, Sun P,Liu Y, Xu Z and Ju J: Downregulation of human intercellular adhesion molecule-1 attenuates the metastatic ability in human breast cancer cell lines. Oncol Rep 35: $1541-1548,2016$

48. Garcia-Palacios V, Chung HY, Choi SJ, Sarmasik A, Kurihara N, Lee JW, Galson DL, Collins R and Roodman GD: Eosinophil chemotactic factor-L (ECF-L) enhances osteoclast formation by increasing in osteoclast precursors expression of LFA-1 and ICAM-1. Bone 40: 316-322, 2007.

49. Tsai CF, Chen JH, Wu CT, Chang PC, Wang SL and Yeh WL: Induction of osteoclast-like cell formation by leptin-induced soluble intercellular adhesion molecule secreted from cancer cells. Ther Adv Med Oncol 11: 1758835919846806, 2019.

50. Bhatia V, Cao Y, Ko TC and Falzon M: Parathyroid hormone-related protein interacts with the transforming growth factor- $\beta$ /bone morphogenetic protein-2/gremlin signaling pathway to regulate proinflammatory and profibrotic mediators in pancreatic acinar and stellate cells. Pancreas 45: 659-670, 2016.

51. Milde-Langosch K, Röder H, Andritzky B, Aslan B, Hemminger G, Brinkmann A, Bamberger CM, Löning T and Bamberger AM: The role of the AP-1 transcription factors c-Fos, FosB, Fra-1 and Fra-2 in the invasion process of mammary carcinomas. Breast Cancer Res Treat 86: 139-152, 2004

52. Chen SP, Liu BX, Xu J, Pei XF, Liao YJ, Yuan F and Zheng F: MiR-449a suppresses the epithelial-mesenchymal transition and metastasis of hepatocellular carcinoma by multiple targets. BMC Cancer 15: 706, 2015.

53. Kwak SC, Baek JM, Lee CH, Yoon KH, Lee MS and Kim JY: Umbelliferone prevents lipopolysaccharide-induced bone loss and suppresses RANKL-induced osteoclastogenesis by attenuating Akt-c-Fos-NFATc1 signaling. Int J Biol Sci 15: 2427-2437, 2019.

54. Berry JE, Ealba EL, Pettway GJ, Datta NS, Swanson EC, Somerman MJ and McCauley LK: JunB as a downstream mediator of PTHrP actions in cementoblasts. J Bone Miner Res 21: 246-57, 2006

55. Rehman AO and Wang CY: CXCL12/SDF-1 alpha activates NF-kappaB and promotes oral cancer invasion through the Carma3/Bcl10/Malt1 complex. Int J Oral Sci 1: 105-118, 2009.

56. Yang F, Takagaki Y, Yoshitomi Y, Ikeda T, Li J, Kitada M, Kumagai A, Kawakita E, Shi S, Kanasaki K and Koya D: Inhibition of dipeptidyl peptidase-4 accelerates epithelial-mesenchymal transition and breast cancer metastasis via the CXCL12/CXCR4/mTOR axis. Cancer Res 79: 735-746, 2019.

57. Shima K, Kimura K, Ishida M, Kishikawa A, Ogawa S, Qi J, Shen WR, Ohori F, Noguchi T, Marahleh A and Kitaura H: C-X-C motif chemokine 12 enhances lipopolysaccharide-induced osteoclastogenesis and bone resorption in vivo. Calcif Tissue Int 103: 431-442, 2018

58. Lei Y, He X, Huang H, He Y, Lan J, Yang J, Liu W and Zhang T: Nerve growth factor orchestrates NGAL and matrix metalloproteinases activity to promote colorectal cancer metastasis. Clin Transl Oncol 24: 34-47, 2022.

59. Lin C, Ren Z, Yang X, Yang R, Chen Y, Liu Z, Dai Z, Zhang Y, He Y, Zhang C, et al: Nerve growth factor (NGF)-TrkA axis in head and neck squamous cell carcinoma triggers EMT and confers resistance to the EGFR inhibitor erlotinib. Cancer Lett 472: 81-96, 2020.

60. Gaspersic R, Kovacic U, Glisovic S, Cör A and Skaleric U: Anti-NGF treatment reduces bone resorption in periodontitis. J Dent Res 89: 515-520, 2010

This work is licensed under a Creative Commons Attribution-NonCommercial-NoDerivatives 4.0 International (CC BY-NC-ND 4.0) License. 